\title{
MS10-05 | Stereochemical Restraints for Nucleic Acids Revisited
}

Jaskolski, Mariusz (A. Mickiewicz University, Poznan, POL); Brzezinski, Dariusz (Poznan University of Technology, Poznan, POL); Kowiel, Marcin (Institute of Bioorganic Chemistry, Polish Academy of Sciences, Poznan, POL); Gilski, Miroslaw (A. Mickiewicz University, Poznan, POL); Zhao, Jianbo (University of Rochester, Rochester, USA); Turner, Douglas (University of Rochester, Rochester, USA)

The reference dictionary of nucleic-acid stereochemistry was compiled (using CSD) in 1996, as presented by Parkinson et al. 23 years later and with 10x expanded CSD, it is time to re-assess the validity of those standards and to see if more sophisticated paradigms (e.g. conformation-dependent functions) might be used. We based our analysis on rigorous statistical treatment of CSD fragments, divided into three main classes of the principal nucleicacid constituents. The covalent geometry of the phosphodiester group is indeed dependent on conformation, albeit in a discrete way, where the O-P-O bond angles and distances are grouped into six conformational categories. The same clustering results were obtained by manual analysis and by machine-learning algorithms, confirming that artificial intelligence can be successfully applied for the discovery of complicated structural patterns. The nucleobase geometry is not expected to be conformation-dependent but in this class we tested different hypotheses, e.g. if the CSD data correctly represent WC base pairs, or if advanced quantum mechanical (QM) calculations could provide sufficiently accurate restraints. While the QM models are remarkably good, they are still inferior to high-quality CSD data. For the glycosidic moiety, the situation is complex because some of the geometrical parameters (e.g. the glycosidic bond) depend on conformation, while other do not. Our results confirm that the Parkinson library is still valid. However, since there are some parameters that require adjustment, we suggest to use the current library. For convenience, a webserver (http://achesym.ibch.poznan.pl/restraintlib/) has been created to generate restraints for the most popular refinement programs. 